\title{
Note
}

\section{La production spermatique du taureau créole}

\author{
P. BELLOIR, E. LAFORTUNE et D. GAUTHIER * \\ avec la collaboration technique de $\mathrm{H}$. VARo \\ I.N.R.A., Station de Recherches zootechniques \\ Centre de Recherches Antilles-Guyane \\ Domaine Duclos, F 97170 Petit-Bourg, Guadeloupe
}

\begin{abstract}
Résumé
Les caractéristiques de la morphologie testiculaire (longueur, diamètre, poids) et la production spermatique sont décrites après abattage pour 42 taureaux créoles élevés en Guadeloupe dans les exploitations agricoles. Les testicules de ces animaux ont une longueur (L) de $10,6 \pm 1,1 \mathrm{~cm}$, un diamètre antéro-postérieur (D) de $5,7 \pm 0,6 \mathrm{~cm}$ et un poids de $155 \pm 41 \mathrm{~g}$. La quantité journalière de spermatozoïdes produits (QJSP), appréciée par la mesure des réserves intratesticulaires, est de $4,7 \pm 2,010^{9}$, de plus cette quantité est liée aux mensurations testiculaires par la relation : QJSP $=0,56 \mathrm{~L}+1,72 \mathrm{D}-10,99$.

Les effets de certains facteurs de variation (âge et état corporel) de ces paramètres sont aussi analysés.
\end{abstract}

Mots clés : production spermatique, taureau créole.

\section{Introduction}

La production spermatique étudiée pour de nombreuses races de taureaux (revue d'AMANN, 1970) est un des paramètres essentiels de la fertilité des mâles (Fulkerson, Synnot \& Lindsay, 1982). C'est pourquoi, il nous apparaît important d'essayer de la déterminer chez le taureau créole dans les conditions traditionnelles d'élevage de la Guadeloupe et d'en analyser quelques facteurs de variation. De plus, son appréciation étant difficilement réalisable dans les conditions de la pratique autrement que par la mesure du diamètre testiculaire, nous avons voulu établir pour cette race la relation entre la production spermatique et les mensurations testiculaires.

* Adresse actuelle : I.N.R.A., Laboratoire de la Production de Viande, Theix, 63122 Ceyrat (France). 


\section{Matériel et méthodes}

Les testicules de 42 tauraux créoles adultes ont été collectés dans trois abattoirs de Guadeloupe entre le $1^{\text {er }}$ avril et le 15 juillet. Sur l'animal abattu et avant castration, la longueur et le diamètre antéropostérieur de chaque testicule sont mesurés (moyenne de deux mesures) à travers le scrotum à l'aide d'un pied à coulisse. L'âge de chaque animal est estimé d'après la dentition et une note d'état général est attribuée avant abattage ( 1 : très bon état ; 4 : mauvais état, animal maigre).

Après abattage, les testicules sont prélevés sur la carcasse et pesés. La quantité journalière de spermatozoïdes produits (QJSP) est calculée d'après la mesure des réserves testiculaires appréciées selon la méthode d'AMANN \& LAMBiAse (1966) et adaptée pour le taureau par Wildeus \& EnTwistle (1982).

Trente grammes de tissus sont prélevés sur chaque testicule et broyés dans du sérum physiologique contenant 0,05 p. 100 de Triton X-100. Le volume final de l'homogénat est, après le broyage, ramené à $500 \mathrm{ml}$. Le nombre de spermatides résistant au broyage est alors compté au microscope à contraste de phase à l'aide d'un hématimètre Thomas. Nous avons cependant testé l'influence du temps de broyage (30', 1', 2', 4', 8') sur le nombre de spermatides comptées pour 4 échantillons de $30 \mathrm{~g}$ prélevés sur le même testicule. L'effet de l'échantillonnage n'est pas significatif alors que celui du temps de broyage est hautement significatif (tabl. 1). Le nombre de spermatides comptées étant le plus élevé après deux minutes de broyage, nous avons retenu ce temps pour le reste de l'expérience.

\section{Tableau 1}

Analyse de variance des effets temps de broyage et numéro d'échantillon sur la quantité de spermatides dénombrées.

Analysis of variance concerning the effects of homogenization time and sample number on the spermatid count.

\begin{tabular}{|c|c|c|c|}
\hline $\begin{array}{l}\text { Sources de variation } \\
\text { Sources of variation }\end{array}$ & ddl & SCE & $\begin{array}{l}\text { Niveau signification } \\
\text { Significance level }\end{array}$ \\
\hline $\begin{array}{l}\text { Temps de broyage } . . . . \\
\text { Homogenization time }\end{array}$ & 4 & 2351,30 & $P<0,01$ \\
\hline $\begin{array}{l}\text { Echantillon } \\
\text { Sample }\end{array}$ & 3 & 162,00 & $P>0,05$ \\
\hline $\begin{array}{l}\text { Erreur } \\
\text { Error }\end{array}$ & 12 & 201,50 & \\
\hline Total & 19 & 2714,80 & \\
\hline
\end{tabular}


La QJSP est obtenue en divisant le nombre de spermatides comptées par 5, 11 jours (SAlim \& ENTWistle, 1982) et la quantité journalière de spermatozoïdes produits par gramme de testicule (QJSPG), en divisant QISP par le poids de testicule.

Les mesures relatives aux testicules droits et gauches étant équivalentes (coefficient de régression non différent de 1 et ordonnée à l'origine non différente de 0 ), les résultats présentés sont donc les valeurs moyennes de deux testicules, sauf la QJSP qui est la production totale du mâle. Les effets des différents facteurs sont appréciés par l'analyse de la variance et les relations entre QISP et mensurations testiculaires étudiées par régression multiple progressive (DAGNELIE, 1980).

\section{Résultats}

\section{A. Caractéristiques générales}

Le poids moyen d'un testicule est de $155 \pm 41 \mathrm{~g}(\mathrm{~m}+\sigma)$, sa longueur 10,6 $\pm 1,1 \mathrm{~cm}$, son diamètre antéro-postérieur $5,7 \pm 0,6 \mathrm{~cm}$ et le rapport longueur/diamètre 1,9. La QJSP est 4,7 $\pm 2,010^{9}$ et la QJSPG $15,2 \pm 3,0 \times 10^{6}$.

Le poids du testicule est relié à sa longueur $(L)(r=0,78 ; P<0,01)$, son diamètre (D) $(r=0,91 ; P<0,001)$ et son volume $\left(V=4 / 3 \pi R^{2} L ; r=0,96\right.$; $\mathrm{P}<0,001)$. La QJSP est liée au poids $(\mathrm{r}=0,8 ; \mathrm{P}<0,001)$, il est donc possible d'obtenir une estimation de la QJSP par l'intermédiaire des variables représentant le poids du testicule ( $L, D, V)$.

Ainsi, QJSP $\left(10^{3}\right)=0,56 \mathrm{~L}(\mathrm{~cm})+1,72 \mathrm{D}(\mathrm{cm})-10,99\left(\mathrm{r}^{2}=0,62\right)$, l'écarttype résiduel est de $1,20 \times 10^{9} \mathrm{SPZ} / \mathrm{j}$. L'élimination de l'un des paramètres (D ou $\mathrm{L}$ ) de la régression augmente significativement l'écart type résiduel. De même, QJSP $\left(10^{9}\right)=14,7 \mathrm{~V}-642,8\left(\mathrm{r}^{2}=0,67\right)$, l'écart-type résiduel est alors de $1,15 \times 10^{9} \mathrm{SPZ} /$ jour. Il n'est, toutefois, pas différent du précédent.

\section{B. Effet de l'âge (tabl. 2)}

Ces paramètres varient tous significativement avec l'âge des animaux (tabl. 2) : les dimensions du testicule et la QJSPG augmentent entre « 1 et 3 dents d'adulte » (de 9,7 à $11,1 \mathrm{~cm}$ pour la longueur, de 5,2 à $5,9 \mathrm{~cm}$ pour le diamètre et de 14,9 à $16,510^{6} \mathrm{SPZ} / \mathrm{g}$ pour la QJSPG) puis ne semblent plus varier par la suite. Le poids et la QJSP s'accroissent régulièrement de « 0 à 4 dents d'adulte 》 (de 87 à $186 \mathrm{~g}$ pour le poids et de 2,3 à $6,2 \quad 10^{9} \mathrm{SPZ} /$ jour pour la QJSP).

\section{Effet de l'état corporel (tabl. 3)}

Bien que non significatif sur la QJSPG, il est très marqué sur les autres paramètres. Les dimensions, le poids testiculaires et la QISP diminuent fortement lorsque l'état corporel se détériore, de 11,2 à $10,0 \mathrm{~cm}$ pour la longueur, de 6,1 à $5,3 \mathrm{~cm}$ pour le diamètre, de 185 à $135 \mathrm{~g}$ pour le poids et de 6,0 à $3,810^{9} \mathrm{SPZ} /$ jour pour la QJSP. 


\section{TABleau 2}

Effet de l'âge apprécié par le développement de la dentition sur quelques paramètres testiculaires du taureau Créole en Guadeloupe.

Effect of age estimated by tooth development on some testicular parameters of Creole bulls in Guadeloupe.

\begin{tabular}{|c|c|c|c|c|c|c|}
\hline $\begin{array}{c}\text { Nombre de dents } \\
\text { d'adultes } \\
\text { Number of adult } \\
\text { teeth }\end{array}$ & $\begin{array}{c}\text { Nombre } \\
\text { d'animaux } \\
\text { Number } \\
\text { of animals }\end{array}$ & $\begin{array}{l}\text { Longueur } \\
\text { (cm) } \\
\text { Length } \\
(\mathrm{cm})\end{array}$ & $\begin{array}{l}\text { Diamètre } \\
(\mathrm{cm}) \\
\text { Diameter } \\
(\mathrm{cm})\end{array}$ & $\begin{array}{l}\text { Poids } \\
\text { (g) } \\
\text { Weight } \\
(g)\end{array}$ & $\begin{array}{c}\text { QJSPG } \\
\cdot \quad\left(10^{6}\right. \\
\times \mathrm{SPZ} / \mathrm{j} / \mathrm{g})\end{array}$ & $\begin{array}{l}\text { QJSP } \\
\left(10^{9}\right. \\
\times \mathrm{SPZ} / \mathrm{j})\end{array}$ \\
\hline 0 & 2 & $10,3^{n}$ & $4,5^{\mathrm{a}}$ & $87 "$ & $13,2^{\mathrm{a}}$ & $2,3^{\mathbf{a}}$ \\
\hline 1 & 9 & $9,7^{\mathrm{lu}}$ & $5,2^{: 1}$ & $131 "$ & $14,9^{a}$ & $3,9^{\mathrm{a}}$ \\
\hline 2 & 11 & $10,3^{a}$ & $5,7^{\prime \prime}$ & $148^{\prime \prime}$ & $12,8^{\prime \prime}$ & $3,8^{\mathrm{a}}$ \\
\hline 3 & 13 & $11,1^{\circ}$ & $5,9^{b}$ & $170^{\circ}$ & $16,5^{1}$ & $5,6^{b}$ \\
\hline 4 & 7 & $11,1^{\circ}$ & $6,0^{\mathrm{b}}$ & $186^{4}$ & 16,711 & $6,2^{\mathrm{b}}$ \\
\hline
\end{tabular}

Les valeurs avec les lettres identiques ne sont pas significativement différentes. Values with the same letters are not significantly different.

TABLEAU 3

Effet de l'état corporel sur quelques paramètres testiculaires du taureau Créole en Guadeloupe.

Effect of body condition on some testicular parameters of Creole bulls in Guadeloupe.

\begin{tabular}{|c|c|c|c|c|c|c|}
\hline $\begin{array}{c}\text { Note d'état } \\
\text { corporel }\end{array}$ & $\begin{array}{c}\text { Nombre } \\
\text { d'animaux }\end{array}$ & $\begin{array}{l}\text { Longueur } \\
\text { (cm) }\end{array}$ & $\begin{array}{l}\text { Diamètre } \\
(\mathrm{cm})\end{array}$ & $\begin{array}{l}\text { Poids } \\
\text { (g) }\end{array}$ & QJSPG & QJSP \\
\hline $\begin{array}{c}\text { Body condition } \\
\text { score }\end{array}$ & $\begin{array}{l}\text { Number } \\
\text { of animals }\end{array}$ & $\begin{array}{l}\text { Length } \\
(\mathrm{cm})\end{array}$ & $\begin{array}{l}\text { Diameter } \\
(\mathrm{cm})\end{array}$ & $\begin{array}{l}\text { Weight } \\
(g)\end{array}$ & $\times \mathrm{SPZ} / \mathrm{j} / \mathrm{g})$ & $\times \mathrm{SPZ} / \mathrm{j})$ \\
\hline 2 & 14 & $11,2^{\mathrm{a}}$ & $6,1^{a}$ & $185^{a}$ & $16,2^{\prime \prime}$ & $6,0^{\mathrm{a}}$ \\
\hline 3 & 21 & $11,3^{\mathrm{a}}$ & $5,5^{b}$ & $141^{b}$ & $14,9^{i}$ & $4,2^{b}$ \\
\hline 4 & 7 & $10,0^{\mathrm{b}}$ & $5,3^{\mathrm{b}}$ & $135^{\mathrm{c}}$ & $14,1^{a t}$ & $3,8^{b}$ \\
\hline
\end{tabular}

Les valeurs avec les lettres identigues ne sont pas significativement différentes.

Values with the same letters are not significantly different. 


\section{Discussion et conclusion}

La morphologie testiculaire du taureau créole (valeur élevée du rapport longueur/diamètre : 1,9) est différente de celle des animaux du genre Bos taurus (1,5, Barba \& Martinez, 1977) et se rapproche de celle des zébus (Bos indicus). Cette particularité semble importante puisque l'introduction de la longueur testiculaire dans la formule de calcul de la production spermatique permet un gain significatif de précision.

La QISPG, élevée par rapport aux résultats obtenus chez les taurins (AMANN, 1970) mais équivalente à celle rapportée pour des zébus (WiLdeus \& ENTWistLe, 1982), ne semble pas provenir d'un artefact puisqu'elle a été confirmée par histologie quantitative (LAFORTUNE, résultats non publiés) sur des animaux dans des conditions contrôlées d'élevage. Toutefois, nos résultats obtenus sur une période courte seraient peut-être à nuancer en fonction de la saison. En effet, les variations de l'offre alimentaire (fourrage), très importantes au cours de l'année, entraînent des changements de niveau nutritionnel et peuvent donc perturber la fonction testiculaire (GAUTHIER \& Berbigier, 1982).

L'évolution, en fonction de l'âge, des paramètres testiculaires étudiés, est le reflet de la croissance des animaux. L'augmentation de volume du testicule, de la QJSP et de la QJSPG se poursuit jusqu'à 2-3 dents alors que celle du poids est plus durable (4 dents). Ces résultats sont comparables à ceux obtenus par WiLdeus \& EnTwistle (1982). L'influence de l'état corporel sur les caractéristiques du testicule traduit les relations qui peuvent exister entre la sous-alimentation et la production de gamètes.

De plus nos résultats montrent que la production spermatique peut être déduite des mensurations testiculaires avec une bonne précision si diamètre et longueur du testicule sont utilisés dans le calcul. L'application de ces mesures de façon systématique avant l'utilisation des mâles en monte naturelle doit permettre d'éliminer les taureauy dont la production spermatique est faible. Toutefois, le nombre de spermatozoïdes produits n'est pas le seul paramètre de la fertilité, il faudrait pouvoir tenir compte de leur qualité et aussi de la libido du mâle.

Reçu en décembre 1983.

Accepté en août 1984.

\section{Summary}

Sperm production of Creole bulls

The testis of 42 Creole bulls, from farms in Guadeloupe were collected at slaughter. Length (L) and diameter (D) were measured through the scrotum. Weight and daily sperm production (DSP) were estimated at the laboratory, the DSP by counting the intratesticular reserves. 
The mean length, antero-posterior diameter and weight were $10.6+1 \mathrm{~cm}(\mathrm{~m} \pm \mathrm{SD})$, $5.7 \pm 0.6 \mathrm{~cm}, 155 \pm 41 \mathrm{~g}$, respectively. The DSP was $4.7 \pm 2.010^{9} \mathrm{SPZ} / \mathrm{day}$ and could be related to the testicular morphology : $\mathrm{DSP}=0.56 \mathrm{~L}+1.72 \mathrm{D}-10.99\left(\mathrm{r}^{2}=0.62\right)$.

The effects of age and body condition score on DSP were analysed.

Key words : sperm production, Creole bull.

\section{R úférences bibliozraphiques}

Amann R.P., Lambiase J.T., 1969. The male rabbit : Ill. - Determination of daily sperm production by means of testicular homogenates. J. Anim. Sci., 28, 369-374.

Amann R.P., 1970. Sperm Production Rates. In the Testis. Johnson A.D., Gomes W.R., Van Demark N.L., eds. Acad. Press. New York. Vol. 1, 433-482.

Barba F., Martinez M., 1977. Desarollo testicular en toros de la raza charolaise. Rev. Cub. Reprod. Anim., 3, 71-75.

Dagnelie P., 1980. Théorie et méthodes statistiques. Vol. 3, eds J. Duculot, S.A. Gembloux.

FulkERSON W.J., SYNNOT A.L., LindSAY D.R., 1982. Number of spermatozoa required to effect a normal rate of conception in naturally mated Merino ewe. Reprod. Fert., 66, 129-132.

Gauthier D., Berbigier P., 1982. The influence of nutritional levels and shade structure on testicular growth and hourly variations of plasma LH and testosterone levels in young creole bulls in a tropical environment. Reprod. Nutr. Develop., 22, 793-801.

Salim B., Entwistle K.W., 1982. Duration of the seminiferous epithelial cycle in hybrid Bos indicus $\times$ Bos taurus bulls. J. Reprod. Fert., 66, 729-734.

Wildeus M.E., Entwistle K.W., 1982. Post puberal changes in gonadal and extragonadal sperm reserves in Bos indicus strain bulls. Theriogenology, 17, 655-667. 